EPJ Web of Conferences 41, 02013 (2013)

DOI: $10.1051 /$ epjconf/20134102013

(C) Owned by the authors, published by EDP Sciences, 2013

\title{
Time-resolved Four-body Coulomb Explosion Imaging of Correlated Dynamics of Hydrogen Atoms in Acetylene Dication
}

\author{
A. Matsuda ${ }^{1}$, M. Fushitani ${ }^{1}$, E.J. Takahashi ${ }^{2}$, and A. Hishikawa ${ }^{1,3}$ \\ ${ }^{1}$ Department of Chemistry, Graduate School of Science, Nagoya University, Furo-cho, Chikusa, \\ Nagoya, Aichi 464-8602, Japan \\ ${ }^{2}$ RIKEN Advanced Science Institute, 2-1 Hirosawa, Wako, Saitama 351-0198, Japan \\ ${ }^{3}$ Institute for Molecular Science, National Institutes of Natural Sciences, Myodaiji, Okazaki, Aichi \\ 444-8585, Japan
}

\begin{abstract}
The correlated motion of the two deuterium atoms associated with the hydrogen migration and structural deformation to non-planar geometry are identified in acetylene dication by the time-resolved four-body Coulomb explosion imaging.
\end{abstract}

\section{Introduction}

Recent advances in laser technology enabled us to utilize few cycle intense laser pulses $(<10 \mathrm{fs}, \sim 1$ $\mathrm{PW} / \mathrm{cm}^{2}$ ) as a new tool for probing ultrafast structural deformation of polyatomic molecules during chemical reaction. Molecules irradiated with such intense laser pulses promptly eject several electrons to form highly charged states, which then undergo rapid bond breaking process by strong Coulombic repulsion between the constituent atoms (ions). The "Coulomb explosion" process provides a unique means to visualize the instantaneous structure of the reaction intermediates, because the momenta of the resultant fragment ions reflect sensitively the geometrical structure of the target molecule at the time of the laser irradiation $[1,2]$.

Hydrogen migration is one of the most important molecular rearrangement process observed in variety of chemical reactions [3]. Isomerization of neutral acetylene and its ionic species between acetylene $(\mathrm{HCCH})$ and vinylidene $\left(\mathrm{H}_{2} \mathrm{CC}\right)$ has been extensively studied as a prototype of hydrogen migration [3-6]. We previously reported the visualization of acetylene dication [5] by pump-probe time-resolved three-body Coulomb explosion imaging. In this study, we extend our previous study on ultrafast hydrogen migration by using time-resolved four-body Coulomb explosion imaging to clarify the correlated motion of the two deuterium atoms in deuterated acetylene dication [7].

\section{Experimental}

A pair of ultrashort intense laser pulses was employed as the pump and probe pulses (Fig. 1(a)). The pump laser pulse was used to doubly ionize $\mathrm{C}_{2} \mathrm{D}_{2}$ to $\mathrm{C}_{2} \mathrm{D}_{2}{ }^{2+}$, and a portion of electronically excited $\mathrm{C}_{2} \mathrm{D}_{2}{ }^{2+}$ undergo hydrogen migration by the strong interaction with the intense laser fields. The probe pulse introduced after a time delay $\Delta t$ prompts the full-fragmentation four-body Coulomb explosion, $\mathrm{C}_{2} \mathrm{D}_{2}{ }^{4+} \rightarrow \mathrm{D}^{+}+\mathrm{C}^{+}+\mathrm{C}^{+}+\mathrm{D}^{+}$, which was used to monitor the instantaneous structure during the hydrogen

This is an Open Access article distributed under the terms of the Creative Commons Attribution License 2.0, which permits unrestricted use, distribution, and reproduction in any medium, provided the original work is properly cited. 

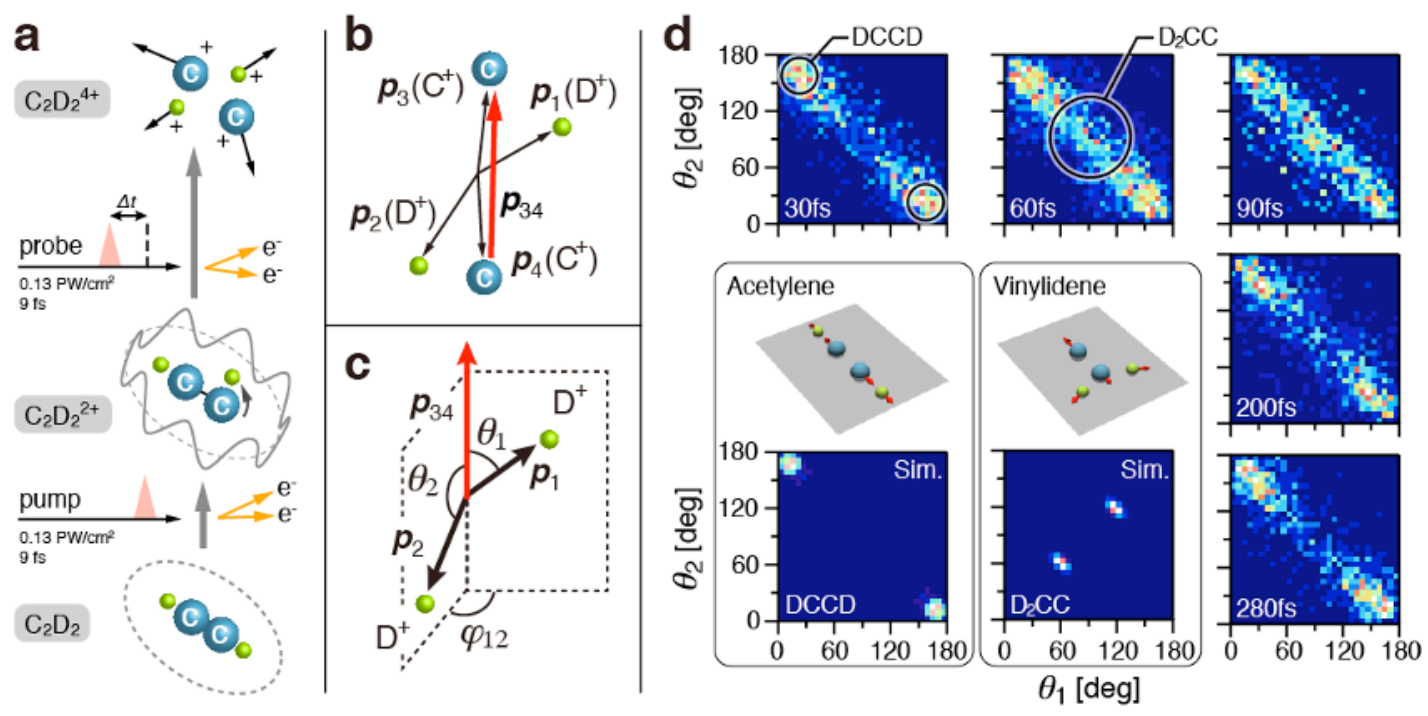

Fig. 1. (a) Scheme of pump-probe Coulomb explosion imaging employed in the present study. (b) Definition of the momenta of the fragment ions and the difference momentum $\boldsymbol{p}_{34}$. (c) Definition of the polar $\left(\theta_{1}, \theta_{2}\right)$ and azimuthal $\left(\varphi_{12}\right)$ angles. (d) Two-dimensional correlation map between $\theta_{1}$ and $\theta_{2}$ at $\Delta t=30,60,90,200$, and 280 fs. The correlation map obtained by the simulation of the four-body Coulomb explosion for typical acetylene (DCCD) and vinylidene $\left(\mathrm{D}_{2} \mathrm{CC}\right)$ structure is also shown.

migration. Linearly polarized Ti:Sapphire laser pulses ( $9 \mathrm{fs}, 800 \mathrm{~nm}, 1 \mathrm{kHz}, 0.4 \mathrm{~mJ} /$ pulse), generated by a pulse compressor consisting of a hollow fiber filled with Ar and a pair of chirp mirrors, was introduced to a high-precision Michelson-type interferometer to generate the pump and probe pulses, which were then focused onto an effusive molecular beam of $\mathrm{C}_{2} \mathrm{D}_{2}$ in an ultrahigh vacuum chamber. The laser field intensities were $0.13 \mathrm{PW} / \mathrm{cm}^{2}$. The momenta of the fragment ions produced by fourbody Coulomb explosion were measured by coincidence momentum imaging method [8].

In order to discuss the correlated motion of the two deuterium atoms in the molecular frame, we utilize the difference momentum of the two $\mathrm{C}^{+}$ions, $\boldsymbol{p}_{34}=\boldsymbol{p}_{3}-\boldsymbol{p}_{4}$, which approximately represents the direction of the C-C bond (Fig. 1(b)). The angles $\theta_{1}$ and $\theta_{2}$ between $\boldsymbol{p}_{34}$ and the momentum of the respective $\mathrm{D}^{+}$ions represent the angular positions of the deuterium atoms in the molecular frame, and the angle $\varphi_{12}$ between the planes spanned by $\boldsymbol{p}_{34}$ and $\boldsymbol{p}_{1}, \boldsymbol{p}_{2}$ represents the azimuthal correlation of the two deuterium atoms (Fig. 1(c)).

\section{Results and discussion}

The experimental correlation map of the momentum angles $\theta_{1}$ and $\theta_{2}$ is shown in Fig. 1(d). At a small time delay $(\Delta t=30 \mathrm{fs})$, the distribution is observed in the regions around $\left(\theta_{1}, \theta_{2}\right)=\left(0^{\circ}, 180^{\circ}\right)$ and $\left(180^{\circ}, 0^{\circ}\right)$. This corresponds to the ejection of $\mathrm{D}^{+}$ions in the opposite directions along the $\mathrm{C}-\mathrm{C}$ bond showing that the two deuterium atoms are located close to their original carbon site and the acetylene structure is preserved. As the time delay increases to $\Delta t=90 \mathrm{fs}$, a new feature appears around $\theta_{1}=\theta_{2}=90^{\circ}$, which indicates that the deuterium atoms shifted their position from the original carbon site towards the other.

At a longer time delay ( $\Delta t=280 \mathrm{fs})$, the new feature disappears and the distributions at $\left(\theta_{1}\right.$, $\left.\theta_{2}\right)=\left(0^{\circ}, 180^{\circ}\right)$ and $\left(180^{\circ}, 0^{\circ}\right)$ revives. This shows that the isomerization occurs in $90 \mathrm{fs}$, followed by further migration of the deuterium atom back to the original acetylene structure in $280 \mathrm{fs}$, which agree well with our previous result obtained using three-body Coulomb explosion imaging [5]. The 
strong distribution along the diagonal line shows that the increase of $\theta_{1}$ from $0^{\circ}$ is always accompanied by the decrease of $\theta_{2}$ from $180^{\circ}$ and vice versa, which clearly indicates that the positions of the two hydrogen atoms are strongly correlated during the migration; when one deuterium atom starts moving, the other deuterium also starts moving from the other carbon site.

A closer inspection of the $\theta_{1}-\theta_{2}$ correlation map provides a more detailed picture. The new feature observed in the $\theta_{1}-\theta_{2}$ correlation map at $\left(\theta_{1}, \theta_{2}\right)=\left(90^{\circ}, 90^{\circ}\right)$ does not exactly appear at the positions expected for a typical vinylidene structure obtained by the simulation of the four-body explosion (Fig. 1(d)). A key to understand the deviation can be found in the distribution of the azimuthal angle $\varphi_{12}$ defined in Fig. 1(c). The time evolution of $\varphi_{12}$ shows that the distribution has a longer tail towards $\varphi_{12}=0^{\circ}$ at $\Delta t=90 \mathrm{fs}$ than those at $\Delta t=30$ and $280 \mathrm{fs}$ (not shown). This indicates that there exists a significant contribution from non-planar structures, showing that hydrogen migration occur in a broad structural distribution containing non-planar geometries.

\section{Conclusion}

The ultrafast hydrogen migration in acetylene dication is studied by means of time-resolved Coulomb explosion imaging with 9 fs intense laser pulses. The four-body, full fragmentation process clarified the strong correlated motion of the hydrogen atoms during the migration process, and besides, the structural deformation to non-planar geometries is revealed. The direct visualization of ultrafast molecular dynamics will not only provide deep insights into the chemical reactions but also offer a new prospect on efficient coherent reaction control with tailored pulses.

\section{References}

1. F. Légaré, K.F. Lee, I.V. Litvinyuk, P.W. Dooley, S.S. Wesolowski, P.R. Bunker, P. Dombi, F. Krausz, A.D. Bandrauk, D.M. Villeneuve, P.B. Corkum, Phys. Rev. A 71, 013415 (2005)

2. A. Matsuda, E.J. Takahashi, A. Hishikawa, J. Chem. Phys. 127, 114318 (2007)

3. H.F. Schaefer III, Acc. Chem. Res. 12, 288 (1979)

4. T. Osipov, C.L. Cocke, M.H. Prior, A. Landers, T. Weber, O. Jaguzki, L. Schmidt, H. SchmidtBöcking, R. Dörner, Phys. Rev. Lett. 90, 233002 (2003)

5. A. Hishikawa, A. Matsuda, M. Fushitani, E.J. Takahashi, Phys. Rev. Lett. 99, 258302 (2007)

6. Y.H. Jiang, A. Rudenko, O. Herrwerth, L. Foucar, M. Kuuka, K.U. Kühnel, M. Lezius, M.F. Kling, J. van Tilborg, A. Belkacem, K. Ueda, S. Düsterer, R. Treusch, C.D. Schröter, R. Moshammer, J. Ullrich, Phys. Rev. Lett. 105, 263002 (2010)

7. A. Matsuda, M. Fushitani, E.J. Takahashi, A. Hishikawa, Phys. Chem. Chem. Phys. 13, 8697 (2011)

8. H. Hasegawa, A. Hishikawa, and K. Yamanouchi, Chem. Phys. Lett. 349, 57 (2001) 